\title{
TRADUÇÃO E ADAPTAÇÃO TRANSCULTURAL DO INSTRUMENTO EVALUATION OF SIDERAIL USAGE
}

\author{
TRANSLATION AND CROSS-CULTURAL ADAPTATION OF \\ THE EVALUATION OF SIDERAIL USAGE INSTRUMENT
}

\section{TRADUCCIÓN Y ADAPTACIÓN TRANSCULTURAL DEL INSTRUMENTO EVALUATION OF SIDERAIL USAGE}

\author{
Teresa Cristina Brasil Ferreira ${ }^{1}$ \\ Rosimere Ferreira Santana ${ }^{2}$ \\ Thalita Gomes do Carmo ${ }^{3}$ \\ Márcia Veríssimo de Souza ${ }^{4}$ \\ Priscilla Alfradique de Souza ${ }^{5}$
}

Como citar este artigo: Ferreira TCB, Santana RF, Carmo TG, Souza MV, Souza PA. Tradução e adaptação transcultural do instrumento Evaluation of Sideral Usage. Rev baiana enferm. 2021;35:e43031.

\begin{abstract}
Objetivo: traduzir e adaptar transculturalmente o instrumento Evaluation of Siderail Usage para a língua portuguesa do Brasil. Método: pesquisa metodológica de tradução e adaptação transcultural de um instrumento em quatro etapas. Resultados: na primeira etapa foram realizadas duas traduções; na segunda, foi realizada uma reunião consensual com duas tradutoras e as autoras do estudo para discutir discrepâncias e gerar uma versão síntese; na terceira etapa, a versão síntese foi retrotraduzida para o inglês por duas tradutoras para se verificar a equivalência com a versão original; e na quarta etapa, um comitê de 25 juízes profissionais da área de saúde analisaram a versão síntese. Os resultados demostraram um Índice de Validade de Conteúdo do instrumento de 0,97 e um Coeficiente de Validade de Conteúdo de 0,93. Conclusão: a tradução e adaptação do instrumento Evaluation of Siderail Usage para a língua portuguesa do Brasil apresentou bons índices de validade do conteúdo.
\end{abstract}

Descritores: Leito. Quedas. Segurança do Paciente. Restrição Física.

Objective: to translate and adapt cross-culturally the Evaluation of Side Rail Usage instrument to the Brazilian Portuguese. Method: methodological research with four-step translation and cross-cultural adaptation of an instrument. Results: in the first stage, two translations were performed; in the second, a consensual meeting was held with two translators and the authors of the study to discuss discrepancies and generate a synthesis version; in the third stage, the synthesis version was backtranslated into English by two translators to verify equivalence with the original version; and in the fourth stage, a committee of 25 professional judges from the bealth area analyzed the synthesis version. The results showed an instrument Content Validity Index of 0.97 and a Content Validity Coefficient of 0.93. Conclusion: the translation and adaptation of the Evaluation of Side Rail Usage instrument into Brazilian Portuguese presented good content validity indexes.

Descriptors: Bed. Falls. Patient Safety. Physical Restriction.

\footnotetext{
Enfermeira. Mestre em Enfermagem Assistencial. Presidente do Núcleo de Segurança do Paciente do Hospital Municipal Miguel Couto. Niterói, Rio de Janeiro, Brasil. http://orcid.org/0000-0002-4780-0115.

Enfermeira. Doutora em Enfermagem. Professora Associada da Universidade Federal Fluminense. Niterói, Rio de Janeiro, Brasil. http://orcid.org/0000-00024593-37I5

3 Enfermeira. Doutora em Ciências do Cuidado da Saúde. Professora Adjunta da Universidade Federal Fluminense. Niterói, Rio de Janeiro, Brasil. thalitacarmo@id.uff.br. http://orcid.org/0000-0002-5868-667X.

Enfermeira. Mestre em Enfermagem Assistencial. Membro da Comissão Terapêutica de Feridas do Hospital Federal dos Servidores do Estado. Niterói, Rio de Janeiro, Brasil. http://orcid.org/0000-000I-9627-9824.

5 Enfermeira. Doutora em Enfermagem. Professora Adjunta da Universidade Federal do Estado do Rio de Janeiro. Rio de Janeiro, Rio de Janeiro, Brasil. http://orcid. org/0000-0002-4625-7552
} 
Objetivo: traducir y adaptar el instrumento Evaluation of Side Rail Usage al idioma portugués brasileño. Método: investigación metodológica de la traducción y adaptación transcultural de un instrumento en cuatro pasos. Resultados: en la primera etapa, se realizaron dos traducciones; en la segunda, se realizó una reunión consensuada con dos traductores y los autores del estudio para discutir discrepancias y generar una versión de sintesis; en la tercera etapa, la versión de sintesis fue retrotraducida al inglés por dos traductores para verificar la equivalencia con la versión original; y en la cuarta etapa, un comité de 25 jueces profesionales del área de salud analizó la versión de sintesis. Los resultados mostraron un índice de validez de contenido del instrumento de 0,97y un Coeficiente de Validez de Contenido de 0,93. Conclusión: la traducción y adaptación del instrumento de Evaluation of Side Rail Usage al portugués brasileño presentó buenos índices de validez de contenido.

Descriptores: Cama. Caídas. Seguridad del Paciente. Restricción Física.

\section{Introdução}

Estudos demonstram que as quedas em pacientes hospitalizados estão associadas ao aumento do uso indiscriminado de grades laterais do leito. Além disso, o uso das grades proporciona gravidade nos eventos adversos relacionados a queda ${ }^{(1)}$. Ainda, eventos adversos aumentam substancialmente as despesas com os cuidados de saúde, estimando-se entre 13 e 16\% dos custos hospitalares ${ }^{(2)}$.

A segurança do paciente é uma política e prática instituída para redução dos riscos de danos desnecessários associados à assistência e à saúde ${ }^{(3)}$. No Brasil, estudo realizado em quatro hospitais, sendo três públicos e um da rede suplementar, evidenciou prevalência de eventos adversos de $12,8 \%$, dos quais $42,7 \%$ foram considerados evitáveis ${ }^{(4)}$.

Os eventos adversos são incidentes que resultam em danos à saúde e são também definidos como injúrias não intencionais decorrentes do cuidado prestado aos pacientes, não relacionadas à evolução natural da doença de base, e que, obrigatoriamente, acarretam lesões mensuráveis nos pacientes afetados, óbito ou prolongamento do tempo de internação ${ }^{(1)}$.

Desde 2004, o tema segurança do paciente vem sendo desenvolvido sistematicamente pela Agência Nacional de Vigilância Sanitária (ANVISA), e tem sido reforçado com a publicação das diretrizes estabelecidas pelo Programa Nacional de Segurança do Paciente (PNSP). Para institucionalizar as práticas seguras, foi determinado aos estabelecimentos de saúde que construíssem os planos locais de segurança do paciente mediante a formação de Comissões, que são os Núcleos de Segurança do Paciente. Dentre os protocolos citados, destacam-se as quedas que resultam em danos e são um problema prevalente de segurança do paciente ${ }^{(5)}$.

Medidas de contenção têm sido utilizadas para prevenir quedas e controlar pacientes com distúrbio de comportamento ou motor. Para permitir a continuidade do tratamento no ambiente hospitalar, a contenção mecânica tem sido realizada principalmente pelo uso de grades para evitar quedas ${ }^{(6)}$.

Estudo observou que a maioria das quedas notificadas foi causada por perda de equilíbrio (26,92\%), desorientação (17,31\%), falha/mau uso de equipamento $(17,31 \%)$ e fraqueza $(15,38 \%)^{(7)}$.

Grades laterais do leito também são consideradas uma forma de contenção quando restringem a liberdade de movimento e impedem que a pessoa saia da cama quando desejar, independentemente da sua capacidade de fazer isto com segurança. As grades têm sido correlacionadas com eventos adversos que incluem agitação, incontinência urinária e fecal, danos e mortes por aprisionamento ${ }^{(8)}$.

Evidências na literatura sugerem que as grades não deveriam ser usadas como contenção mecânica, entretanto, esse fato tem sido ignorado com frequência, comprometendo a segurança, a dignidade e a autonomia do paciente ${ }^{(9)}$.

Os danos são mais frequentes e ocorrem quando os pacientes tentam escalar as grades para sair da cama ${ }^{(10)}$. O aumento do uso de grades não resultou em diminuição e recorrência 
no número de quedas, mas aumentou a queda com ferimentos graves.

O presente estudo tem por objetivo traduzir e adaptar transculturalmente o instrumento Evaluation of Siderail Usage ${ }^{(9)}$ para a língua portuguesa do Brasil.

\section{Método}

Trata-se de pesquisa metodológica de tradução e adaptação transcultural do instrumento Evaluation of Siderail Usage com abordagem quantitativa.

O instrumento Evaluation of Siderail Usage foi desenvolvido nos Estados Unidos (US) para avaliar o uso de grades de proteção em instituições de longa permanência. Subdivide-se em quatro partes, a saber: preferência do paciente (ou residente); determinação do risco de danos (por exemplo, histórico de quedas e presença de contusões e lesões causadas pelas grades); risco de queda da cama (que considera fatores, como dificuldade de mobilidade, equilíbrio, confusão mental, dentre outros); e avaliação de alternativas (recomendações para que se reduza a necessidade de utilizar contenção e grades).

A escolha do instrumento deu-se pela livre disponibilização por via eletrônica, sem ônus, e pela possibilidade de usá-lo em diferentes contextos culturais. Para iniciar o processo de tradução e adaptação transcultural foi necessário contatar a autora principal, Dra. Elizabeth Capezuti, por correio eletrônico, para obter a sua autorização. A autora permitiu que fosse realizada a pesquisa no Brasil e os resultados obtidos foram submetidos ao consentimento da autora.

O estudo seguiu as seguintes etapas: tradução do instrumento, síntese das versões, retrotradução (back translation) e revisão da síntese por experts ${ }^{(11-12)}$.

\section{Etapa 1 - Tradução do instrumento}

A tradução de um instrumento do idioma de origem para o idioma-alvo é um processo complexo, que exige alguns cuidados, para obter-se uma versão final adequada para o novo contexto e não modifique as características da versão original.

Sugere-se que a tradução seja realizada por, pelo menos, dois tradutores bilíngues, minimizando o risco de vieses linguísticos, psicológicos, culturais e de compreensão ${ }^{(12)}$.

Foi realizada a tradução da versão original do instrumento do inglês para o português por duas tradutoras bilíngues, separadamente, que tinham como língua materna o português do Brasil, com fluência no idioma inglês. Em seguida, surgiram as duas versões $\mathrm{T}_{1}$ e $\mathrm{T}_{2}$.

Uma das tradutoras selecionadas é representante da área da saúde, portanto, tinha conhecimento sobre a temática. A outra tradutora era formada em Letras e não possuía conhecimento técnico acerca do assunto. Nesta etapa, cada uma das tradutoras elaborou uma versão do instrumento.

\section{Etapa 2 - Sintese das versões}

De posse de ambas as traduções realizadas, criou-se a síntese das versões. Para tanto, as duas traduções foram comparadas quanto à existência de discrepâncias semânticas, idiomáticas, conceituais, linguísticas e contextuais. De modo presencial, os pesquisadores e as duas tradutoras realizaram a síntese das duas traduções $T_{1}$ e $\mathrm{T}_{2}$, resultando na versão $\mathrm{T}_{12}$ após reunião de consenso.

\section{Etapa 3-Retrotradução (back translation)}

Após a nova versão traduzida e sem a influência da versão original, o instrumento foi traduzido de volta para o idioma original (inglês). Esta etapa é necessária para garantir a validade do instrumento e para que ele reflita, precisamente, o conteúdo da versão original.

Duas retrotraduções foram realizadas por tradutores bilíngues que possuem o idioma inglês como língua materna. Os retrotradutores não devem estar informados acerca do assunto abordado no instrumento e, preferencialmente, não devem ser da área da saúde. Dessa forma, evitou-se viés de informação e que 
surgissem significados inesperados dos itens do instrumento $^{(14-15)}$.

Neste estudo, a versão síntese do instrumento em português foi retrotraduzida para o inglês por duas tradutoras independentes, estrangeiras, que têm o inglês como língua materna e fluência no português. As tradutoras não participaram da tradução inicial, não tiveram acesso à versão original do instrumento e não foram informadas do objetivo da pesquisa.

Verificou-se, nessa etapa, se a versão em português refletia o conteúdo da versão original, resultando na versão em inglês do instrumento construída pelos tradutores, individualmente. Essa versão foi enviada à autora da escala para avaliação.

\section{Etapa 4-Revisão da sintese por experts}

Nesta etapa foi realizada a equivalência transcultural do instrumento por um comitê de experts. Todas as versões do instrumento, incluindo instruções, foram consolidadas, e obteve-se a versão pré-teste. As discrepâncias encontradas foram resolvidas por consenso.

O comitê avaliou a equivalência entre as versões traduzidas e o instrumento original em cada uma das seguintes áreas: semântica, idiomática, experiencial e conceitual.

O comitê de experts foi composto por enfermeiros, profissionais de saúde e estudiosos da área de contenção que atenderam a, pelo menos, dois dos seguintes critérios: experiência mínima de três anos no ensino ou na prática de Contenção mecânica, Prevenção de quedas, e Segurança do Paciente; domínio dos idiomas português e inglês; domínio de metodologia de pesquisa; e ter participado de pesquisas na área da enfermagem e na área de construção e validação de escalas e instrumentos.

Frente à inexistência de uma recomendação referente ao número de experts para o tipo de validação pretendida, estipulou-se um número de 30 participantes (número mínimo indicado para análises estatísticas). Os experts foram selecionados de forma presencial ou por correio eletrônico após pesquisa no currículo Lattes.
Aceitaram participar da pesquisa 25 profissionais de saúde. Considerou-se a experiência e a qualificação no assunto estudado.

Os experts participaram de um encontro para apresentação da pesquisa, preenchimento de um formulário de avaliação do instrumento em inglês e português, individualmente, e esclarecimento de dúvidas. No formulário utilizou-se uma escala do tipo Likert, com as seguintes opções: (1) discordo totalmente, (2) discordo parcialmente, (3) concordo parcialmente, (4) concordo totalmente, e um espaço para observações e sugestões.

\section{Análise dos dados}

Para a análise dos dados, foi construído um banco de dados por meio do programa Microsoft Excel 2013 e foram realizadas análises estatísticas por meio do programa Statiscal Package for the Social Sciences (SPSS), versão 22.0.

Para caracterização da amostra dos juízes, foi usada a estatística descritiva baseada em gráficos, distribuição de frequências e cálculo de estatísticas (média, mediana, desvio-padrão, máximo e mínimo, coeficiente de variação) das variáveis quantitativas.

Para validação do conteúdo para cada item e para o instrumento global foi calculado o Índice de Validade de Conteúdo (IVC). O IVC mede a proporção ou porcentagem de juízes que estão em concordância sobre determinados aspectos do instrumento e de seus itens ${ }^{(16)}$.

Além do IVC, foi calculado o Coeficiente de Validação do Conteúdo (CVC) para quantificar e interpretar o julgamento de itens e escalas por um grupo de especialistas no construto que o instrumento propõe medir ${ }^{(16)}$. Os valores de CVC aceitos para considerar a qualidade de um aspecto ou um item julgado devem ser maiores que 0,80 .

Ressalta-se que este estudo foi aprovado pelo Comitê de Ética em Pesquisa, sob o Parecer n. 3.013.114/2018, seguindo as recomendações da Resolução n. 466/12 do Conselho Nacional de Saúde. 


\section{Resultados}

O estudo contou com amostra de 25 juízes, dentre os quais 4 eram do sexo masculino $(16,0 \%)$ e 21 do sexo feminino $(84,0 \%)$. Predominou na amostra juízes-enfermeiros 20 (80,0\%), juízes-médicos $2(8,0 \%)$ e 1 juiz de cada uma das respectivas formações: Direito, Fisioterapia e Pedagogia. A maioria possuía 7 a 11 anos de formação $(39,1 \%)$.

Os juízes avaliaram 40 itens do questionário, respondendo cada item de acordo com uma escala do tipo Likert, para análise do IVC e CVC, apresentada na Tabela 1 .

Tabela 1 - Distribuição dos índices de índice de validade de conteúdo e coeficiente de validade de conteúdo para cada item e global. Rio de Janeiro, Rio de Janeiro, Brasil - 2019 (continua)

\begin{tabular}{|c|c|c|}
\hline Itens & IVC & CVC \\
\hline Paciente/Quarto & 0,92 & 0,87 \\
\hline Preferência do paciente & 1,00 & 0,93 \\
\hline O paciente está apto a expressar sua preferência em relação às grades? & 1,00 & 0,92 \\
\hline $\begin{array}{l}\text { O paciente assinou um termo de consentimento indicando sua preferência sobre } \\
\text { grades laterais? }\end{array}$ & 1,00 & 0,92 \\
\hline Se 2 = sim, quantas grades o paciente prefere? & 1,00 & 0,94 \\
\hline Avaliação de Risco & 0,96 & 0,96 \\
\hline O paciente tem histórico de quedas em geral? & 1,00 & 0,98 \\
\hline O paciente tem histórico de quedas do leito? & 0,96 & 0,96 \\
\hline O paciente faz tentativas de escalar ou passar por entre as grades laterais? & 0,96 & 0,93 \\
\hline $\begin{array}{l}\text { O paciente já ficou aprisionado entre as grades ou entre o colchão e a grade } \\
\text { lateral? }\end{array}$ & 0,96 & 0,96 \\
\hline $\begin{array}{l}\text { O paciente apresenta ou já apresentou hematomas, lesões por fricção ou } \\
\text { laceração provocados por uma grade lateral? }\end{array}$ & 1,00 & 0,89 \\
\hline Risco de Queda do Leito & 1,00 & 1,00 \\
\hline $\begin{array}{l}\text { O paciente está imóvel (em coma/comatoso, paralisado ou sem movimentos } \\
\text { espontâneos)? }\end{array}$ & 1,00 & 0,98 \\
\hline Se imóvel, o paciente se inclina para um dos lados? & 1,00 & 0,93 \\
\hline $\begin{array}{l}\text { Se imóvel (parte inferior do corpo) ou com mobilidade, o paciente usa a grade } \\
\text { para ajustar a posição no leito? }\end{array}$ & 1,00 & 0,96 \\
\hline Se com mobilidade, o paciente faz alguma tentativa de sair do leito? & 1,00 & 0,96 \\
\hline $\begin{array}{l}\text { Se com mobilidade, o paciente pode entrar e sair do leito com segurança sem } \\
\text { qualquer ajuda ou dispositivo de apoio? }\end{array}$ & 1,00 & 0,95 \\
\hline Se com mobilidade, o paciente tem dificuldade para se equilibrar ou controlar o tronco? & 1,00 & 0,94 \\
\hline $\begin{array}{l}\text { Se com mobilidade o paciente apresenta redução da percepção de segurança } \\
\text { devido a problemas cognitivos ou dificuldades de julgamento? }\end{array}$ & 0,96 & 0,89 \\
\hline Considerações ou observações individuais & 1,00 & 0,95 \\
\hline Avaliação adicional & 1,00 & 0,98 \\
\hline Setor/Avaliação & 0,92 & 0,92 \\
\hline $\begin{array}{l}\text { Enfermagem: monitoramento no período noturno dos movimentos do paciente, } \\
\text { com ambas as grades elevadas }\end{array}$ & 0,96 & 0,88 \\
\hline $\begin{array}{l}\text { Enfermagem: monitoramento no período noturno dos movimentos do paciente, } \\
\text { com ou sem uma das grades }\end{array}$ & 0,92 & 0,87 \\
\hline Avaliações multidisciplinares para transferência e/ou capacidade de deambulação & 0,92 & 0,85 \\
\hline Avaliação do uso de grades & 0,96 & 0,97 \\
\hline Avaliação das alternativas & 1,00 & 0,94 \\
\hline Problema & 1,00 & 0,94 \\
\hline Providenciar campainha chamada ao alcance & 0,92 & 0,91 \\
\hline Programar assistência ao banheiro à noite & 0,92 & 0,90 \\
\hline Reduzir o tempo no leito & 1,00 & 0,94 \\
\hline Aumentar a frequência de monitoramento & 0,96 & 0,95 \\
\hline Colocar dispositivos de apoio à beira do leito & 1,00 & 0,95 \\
\hline Cuidados restauradores para aumentar habilidades para se levant & 1,00 & 0,96 \\
\hline
\end{tabular}


Tabela 1 - Distribuição dos índices de índice de validade de conteúdo e coeficiente de validade de conteúdo para cada item e global. Rio de Janeiro, Rio de Janeiro, Brasil - 2019

\begin{tabular}{lcc}
\hline Itens & IVC & CVC \\
\hline Setor/Avaliação & 0,92 & 0,92 \\
Utilizar metade ou um quarto de grade para mobilidade no leito ou para & 1,00 & 0,95 \\
possibilitar a transferência & 1,00 & 0,96 \\
Providenciar travesseiros ou almofadas como demarcadores de limite do leito & 0,96 & 0,95 \\
Providenciar coxim ou protetor acolchoado na grade lateral (Ver item 1 ou 2) & 0,97 \\
Providenciar alarme no leito & 1,00 & 0,98 \\
Manter cama baixa & 1,00 \\
Manter colchonete no chão próximo ao leito (Ver item 1 ou 2) & 1,00 & 0,96 \\
Outros & 1,00 & 0,97 \\
Recomendações do Comitê de Prevenção e Redução de Contenção e uso de & 0,96 & 0,90 \\
grades laterais & 1,00 \\
Nenhuma grade lateral é indicada porque o residente é capaz de entrar e sair do & 0,88 \\
leito de forma segura & 0,96 \\
As grades laterais estão indicadas porque o paciente está imóvel e não faz & 0,93 \\
nenhuma tentativa para sair ou se virar para o lado & 0,96 \\
Uma grade lateral inteira está indicada para ajudar na mobilidade no leito. & 0,91 \\
Indique direita e esquerda & 0,92 \\
Ambas as grades laterais (inteiras) são as contenções menos restritivas. & 0,90 \\
Ambas as grades laterais (inteiras) não são usadas como contenção, pois o & 0,88 & 0,88 \\
paciente está imóvel & 1,00 \\
Alternativa para grades laterais & 0,84 \\
Avaliações multidisciplinares para problemas e/ou manutenção do leito (travas, \\
grade lateral nivelada ao colchão) \\
Assinaturas dos participantes membros do comitê de prevenção e redução de \\
contenção & 1,00 & 1,00 \\
\hline
\end{tabular}

Fonte: Elaboração própria.

Legenda:

IVC: Índice de Validade de Conteúdo.

CVC: Coeficiente de Validade de Conteúdo.

Observou-se um IVC geral de 0,97. O CVC para avaliar o grau concordância dos experts global do estudo foi de 0,93.

Também durante a reunião do comitê dos juízes obteve-se contribuições qualitativas com sugestões para a validação do instrumento apresentado no Quadro 1.

Quadro 1 - Sugestões qualitativas do comitê de juízes. Rio de Janeiro, Rio de Janeiro, Brasil $-2019$

(continua)

\begin{tabular}{|l|l|}
\hline Versão RT & \multicolumn{1}{|c|}{ Sugestões do comitê de juízes } \\
\hline Residente/quarto & Paciente/leito \\
\hline Apto de & Apto a \\
\hline Formulário de preferência & Termo de consentimento informado \\
\hline Nenhuma Anterior, $0,1,2$ & Acrescentar 3 e 4 grades \\
\hline Determinação do risco & Avaliação de risco \\
\hline História & Histórico \\
\hline Passar por cima/em volta & Escalar/entre \\
\hline Aprisionado & Preso \\
\hline Apresenta & Acrescenta ou já apresentou \\
\hline De uma grade/Apresenta & $\begin{array}{l}\text { Provocados por uma grade (retirou-se o termo de } \\
\text { metal/já apresentou }\end{array}$ \\
\hline
\end{tabular}


Quadro 1 - Sugestões qualitativas do comitê de juízes. Rio de Janeiro, Rio de Janeiro, Brasil - 2019

\begin{tabular}{|c|c|}
\hline Versão $\mathbf{R T}_{1-2}$ & Sugestões do comitê de juízes \\
\hline Inclina-se & Se inclina \\
\hline Móvel & Com mobilidade \\
\hline Assistência humana & Ajuda \\
\hline De equilíbrio, controle do tronco & Para se equilibrar ou controlar o tronco \\
\hline $\begin{array}{l}\text { Consciência diminuída de segurança/Confusão/ } \\
\text { problemas de julgamento }\end{array}$ & $\begin{array}{l}\text { Redução da percepção de Segurança/Cognitivos/ } \\
\text { dificuldades de julgamento }\end{array}$ \\
\hline $\begin{array}{l}\text { Preocupações individuais/qualquer coisa/ache/ } \\
\text { útil/grades laterais }\end{array}$ & $\begin{array}{l}\text { Considerações ou observações individuais/Algo/ } \\
\text { pense/que possa ajudar/acrescentar quanto/retirar } \\
\text { laterais }\end{array}$ \\
\hline Departamento/Avaliação & Setor/Unidade \\
\hline Monitoramento & Monitoramento no \\
\hline Uma grade & Uma das grades \\
\hline $\begin{array}{l}\text { Avaliações de terapeuta ocupacional e } \\
\text { fisioterapeuta ou habilidadesde deambulação }\end{array}$ & $\begin{array}{l}\text { Avaliações multidisciplinares/e ou/capacidades de } \\
\text { deambulação }\end{array}$ \\
\hline $\begin{array}{l}\text { Terapeuta ocupacional/fisioterapia/problemas } \\
\text { com equipamento/descreva }\end{array}$ & $\begin{array}{l}\text { Avaliações multidisciplinares/problemas de } \\
\text { manutenção/travas/Indique }\end{array}$ \\
\hline Descreva & Indique \\
\hline Refira aos números & Localize os números \\
\hline Intervenções alternativas & Alternativas de intervenções \\
\hline $\begin{array}{l}\text { Campainha de chamada ao alcance ou } \\
\text { campainha do tipo bulbo }\end{array}$ & Providenciar campainha de chamada ao alcance \\
\hline Programou? & Programar \\
\hline Diminuiu o tempo no leito? & Reduzir o tempo no leito \\
\hline Aumentou? & Aumentar \\
\hline Colocação? & Colocar \\
\hline Cuidados? & Acrescentar instituir cuidados \\
\hline Metade?/permitir & Acrescentar Utilizar metade/possibilitar \\
\hline Travesseiros & Acrescentar Providenciar travesseiros \\
\hline Coxim? & Acrescentar Coxim \\
\hline Leito baixo? & Manter cama baixa \\
\hline Tapete & Colchonete \\
\hline $\begin{array}{l}\text { Restrição/recomendações do comitê de } \\
\text { prevenção e redução de grade no leito }\end{array}$ & $\begin{array}{l}\text { Recomendações do Comitê de prevenção e } \\
\text { redução de contenção e uso de grades laterais }\end{array}$ \\
\hline Barra lateral & Grade lateral \\
\hline $\begin{array}{l}\text { O residente está imóvel e não faz nenhuma } \\
\text { tentativa de sair ou não se inclina para um } \\
\text { lado? }\end{array}$ & $\begin{array}{l}\text { As grades laterais são indicadas porque o paciente } \\
\text { está imóvel ou não faz nenhuma tentativa para sair } \\
\text { ou se virar para o lado }\end{array}$ \\
\hline $\begin{array}{l}\text { Uma grade lateral inteira indicada para auxiliar } \\
\text { na mobilidade no leito? }\end{array}$ & $\begin{array}{l}\text { Uma grade lateral inteira está indicada para auxiliar } \\
\text { na mobilidade no leito? }\end{array}$ \\
\hline Comprimento completo & Inteiras \\
\hline $\begin{array}{l}\text { Ambas as grades laterais de comprimento } \\
\text { completo não são usadas para restringir, pois } \\
\text { o residente está imóvel? }\end{array}$ & $\begin{array}{l}\text { Ambas as grades laterais (inteiras) não são usadas } \\
\text { como contenção porque o paciente está imóvel }\end{array}$ \\
\hline
\end{tabular}

Fonte: Elaboração própria.

Nota:

$\mathrm{RT}_{1-2}$ Retrotradução: primeira e segunda versões. 


\section{Discussão}

O instrumento avaliado permitiu uma abordagem detalhada e abrangente de avaliação dos pacientes quanto ao uso de grades. Requer que o enfermeiro preencha um formulário sobre o estado físico e psicológico do paciente, considerando a organização do ambiente, e apesar de objetivo, demanda algum tempo dos enfermeiros para realizar as investigações. Outro limite do instrumento é que ele não foi projetado para uso repetido, e o enfermeiro deve decidir quando reaplicá-lo. No entanto, estudos futuros temporais podem auxiliar nesse aprofundamento. Além disso, também possui uma avaliação qualitativa, isto é, não categoriza ou dá peso para indicar o uso de grades ${ }^{(10)}$. Isso tanto pode ser positivo quanto dá liberdade de decisão ao enfermeiro.

O estudo demonstra que a tradução e o processo de adaptação cultural produziram um instrumento capaz de auxiliar o enfermeiro na decisão pelo uso de grades laterais na cama, avaliando o menor grau possível de contenção mecânica ao paciente. Leva em consideração a preferência do paciente, o risco de queda/lesão, a mobilidade e a necessidade de grades, além de propor ao enfermeiro possíveis intervenções para o não uso das grades laterais.

De acordo com as sugestões qualitativas dos juízes, alguns termos foram modificados e outros acrescentados. Optou-se por "paciente" no lugar de "residente" e "leito" ao invés de "cama", que são termos mais utilizados na área hospitalar. No item referente à assinatura de formulário de preferência sobre grades, os juízes preferiram utilizar "termo de consentimento informado", que é entendido como uma decisão voluntária, realizada por pessoa autônoma e capaz, após processo informativo e deliberativo, visando a aceitação de tratamento específico ou experimentação, ao possuir conhecimento da sua natureza, das suas consequências e dos seus riscos ${ }^{(18)}$.

No que tange ao item determinação de risco, os juízes optaram apenas pela adequação do termo para avaliação de risco. O construto trata da avaliação dos riscos de maneira geral, aos quais o paciente está submetido ao utilizar as grades, como quedas, lesões de pele e aprisionamento.

Grades podem representar um risco inerente à segurança do paciente, particularmente quando o paciente é idoso e desorientado, uma vez que estes estão suscetíveis a serem aprisionados entre o colchão e a grade, além de deslizarem entre as grades e seus segmentos ou ainda de escorregarem entre o final da cama e as grades laterais levantadas. Evidências indicam que as meias grades e as inteiras representam um risco de aprisionamento, bem como de quedas, pois levam os pacientes a escalarem as grades para sair ${ }^{(19)}$. Ao tentar sair da cama por qualquer uma dessas rotas, o paciente corre o risco de ser aprisionado, ficar engatado ou cair de uma altura maior por causa das grades elevadas, com a possibilidade de sofrer uma lesão maior ou, até mesmo, a morte, se comparado com uma queda resultante de uma cama abaixada, sem grades laterais erguidas.

Quando se tratou do risco de quedas do leito, o item "Se móvel, o residente tem consciência diminuída de segurança devido à confusão ou problemas de julgamento?" foi alterado para "Se móvel, o paciente tem baixa percepção de segurança devido a problemas de confusão (mental) ou julgamento?" O estado mental prejudicado, confusão e desorientação são frequentemente citados como causas de quedas em idosos hospitalizados ${ }^{(20)}$.

A decisão de usar grades na cama deve ser tomada em conjunto com a vontade do paciente, baseando-se nos princípios de cuidado seguro e mitigação da contenção. A avaliação do paciente pela equipe de saúde deverá contemplar: o problema apresentado (a necessidade do uso de grades) e estratégias alternativas; os riscos individuais de danos ao paciente quanto ao uso de grades; e a redução dos danos se for tomada a decisão de usar as grades ${ }^{(21)}$.

No item avaliações de terapeuta ocupacional/fisioterapeuta para transferência ou habilidades de deambulação, os juízes optaram por utilizar avaliações multidisciplinares para transferência e/ou capacidade de deambulação, 
no intuito de ampliar a possibilidade de profissionais habilitados que pudessem realizar essa avaliação.

As sugestões qualitativas dos juízes foram valiosas e auxiliaram a adaptação transcultural do instrumento. Citamos, por exemplo, que em todos os itens que apresentavam a palavra "residente", esta foi substituída por "paciente", já que o instrumento está sendo adaptado para uso na área hospitalar. Já na fase de pré-teste do instrumento, realizado com enfermeiros da prática hospitalar e com pacientes hospitalizados, importou-se observar o entendimento, as dificuldades, as dúvidas acerca do instrumento, a clareza e os resultados que o instrumento é capaz de gerar quando aplicado ao perfil esperado. Sugere-se que, futuramente, o instrumento possa ser testado com uma amostra maior.

Foi solicitado a cada enfermeiro que, antes de utilizar o instrumento, decidisse quantas grades o paciente deveria usar e, em seguida, utilizasse o instrumento no mesmo paciente. Ao medir os coeficientes de concordância entre os itens Quantas grades o paciente prefere? e Avaliação subjetiva do enfermeiro em relação às grades, observou- se que existe concordância, porém fraca.

As maiores correlações foram apresentadas no item "nenhuma grade lateral é indicada e na escolha do paciente por nenhuma grade (zero grade)". Isso ocorre porque esta seria a avaliação com menor grau de discrepância e a mais fácil conceitualmente e clinicamente de ser correlacionada.

Observa-se no perfil dos pacientes que as mulheres possuem maior risco de serem contidas. Isso pode ser explicado devido as mulheres terem maior comprometimento da saúde e idade, como também pela questão de gênero, que merece ser melhor investigada.

Sobre o perfil dos enfermeiros, não houve nenhuma característica que associasse de forma válida a decisão do uso de grades laterais. A formação especialista foi somente a mais comum e provavelmente deu-se ao acaso.

Sobre a análise de constructo, observaram-se alguns valores significativos que, quando confrontados com os valores do índice de validade de conteúdo, auxiliaram na decisão de retirada de itens para a construção do instrumento final. Por último, foi avaliada a praticabilidade do instrumento.

A praticabilidade é um dos critérios que auxilia na avaliação da qualidade de instrumentos. De modo geral, problemas de praticabilidade incluem problemas com a administração, como tempo muito curto disponibilizado para respostas ou número de itens excessivo, instruções complicadas e descrições confusas dos itens, gerando problemas com a interpretação das perguntas e registro na pontuação $^{(20)}$.

Os resultados da praticabilidade da versão brasileira do instrumento "Avaliação do uso de grades" mostraram que os enfermeiros concordaram totalmente que seria interessante contar com esse instrumento na sua prática clínica. Perguntados em relação ao entendimento das instruções de preenchimento, $86,7 \%$ foi de concordância, indicando a necessidade de revisão dos itens. Entretanto, é importante relatar que nenhum enfermeiro marcou no quesito "não tenho opinião", o que demonstra que todos os participantes do pré-teste estavam dispostos a contribuir para a pesquisa.

Importante destacar que a aplicação do instrumento se refere à análise dos riscos e benefícios do uso de grades, com evidências de que cerca de 20,0\% de todas as quedas hospitalares são do leito. Os pacientes têm atitudes positivas em relação ao uso de grades quando necessárias à sua segurança, mas, o uso excessivo de grades, assim como sua abolição sem as devidas avaliações, aumenta o risco de quedas e lesões, tornando o instrumento uma relevante estratégia para tomada de decisão clínica do enfermeiro ${ }^{(22)}$.

Por outro lado, as grades são inadequadas e ineficazes para pacientes que querem sair da cama e podem ser ativamente prejudiciais para pacientes que combinam confusão grave com mobilidade suficiente. Além disso, as grades não são apropriadas para pacientes independentes, com mobilidade, e para pacientes confusos devido ao risco de escalarem as grades ${ }^{(22)}$. 
Os riscos e benefícios do uso de grades devem ser analisados mediante avaliação individual de cada paciente. As grades têm diversas funções incluindo segurança no transporte, conforto e segurança para o paciente, barreira física para relembrar os limites da cama, suporte para mobilização da cama, reposicionamento e ajuda para entrar e sair da cama ${ }^{(22)}$.

É necessária uma avaliação individualizada, o pode ser obtido por meio do instrumento para avaliação da necessidade do uso de grades laterais, proposto neste estudo.

Devem fazer parte dessa avaliação: revisões da história do residente/paciente (estado mental, sono, mobilidade, incontinência, dor, histórico de quedas e lesões), motivos pelos quais os profissionais decidiram sobre o uso de grades, com registro no prontuário; exame físico (estatura para verificar se o paciente tem pernas curtas, peso, problemas de visão, movimentos de amplitude, se tem necessidade de ir ao banheiro à noite, avaliação da dor); características do ambiente cama/banheiro, iluminação; identificar fatores de riscos específicos do paciente ${ }^{(10)}$.

Um plano de cuidados deve ser desenvolvido após essa avaliação. As grades não são indicadas para adultos ou idosos que estão confusos, uma vez que eles não compreendem sua proposta ou quando relatam que as grades os deixam acuados ${ }^{(23)}$.

As grades laterais não são consideradas contenção se elas são usadas em paciente que não está funcionalmente capaz de fazer movimentos voluntários ou involuntários (embora sua função possa ser questionada neste caso); se o paciente deseja usar as grades laterais como uma ajuda na mobilidade (por exemplo, uma grade lateral para ajudar na rotação) e pode fazê-lo com competência; se o paciente que as solicita como uma sugestão de auxílio no ambiente (para lembrar-se de não se levantar sem ajuda) as utiliza para isso.

Por outro lado, a grade é considerada contenção se o paciente está tentando sair da cama ou quer sair e é impedido de fazê-lo pelo uso de ambas as grades laterais (independentemente da competência em mobilidade); se o paciente ou acompanhante insiste em grades laterais por qualquer motivo, mas o paciente tem dificuldade em sair com segurança da cama; ou se a grade impede ou limita o paciente de sair da cama ${ }^{(21)}$. Aqueles que receberam sedação de qualquer tipo e não estão mostrando sinais contínuos de tentativas de deixar a cama sem ajuda, bem como obesos ou em uso de colchão pneumático, também não deveriam usar grades ${ }^{(23)}$.

As grades podem ser utilizadas para pacientes que se debatem na cama decorrente de delirium, epilepsia ou estão em coma induzido. Embora o estado de confusão do paciente não seja uma contraindicação para o uso de grades, a confusão combinada com mobilidade suficiente pode ser um risco de o paciente escalar as grades da cama. Mesmo assim, a maioria das políticas no Reino Unido recomenda avaliação muito cuidadosa para o uso de grades no paciente com distúrbios cognitivos ${ }^{(22)}$.

Este estudo tem relevância para a prática clínica do enfermeiro contribuindo para tomada de decisão baseada no raciocínio/julgamento direcionado para a qualidade da assistência, pautado na melhor evidência prática. Contribui ainda com a Política de Segurança do Paciente na implantação da meta 6 (diminuir os índices de queda), ao produzir um instrumento útil para avaliação e determinação dos riscos inerentes aos paciente relacionados a quedas e ao uso de grades laterais do leito. A limitação do instrumento requer que o enfermeiro preencha um formulário sobre o estado físico e psicológico do paciente considerando a organização do ambiente. Não obstante ser objetivo, demanda algum tempo dos enfermeiros para ser preenchido. Além disso, o instrumento não foi projetado para uso rotineiro, e o enfermeiro deve decidir quando poderá reaplicá-lo. No entanto, estudos futuros temporais podem ajudar nesse aprofundamento. Como também possui uma avaliação qualitativa, não categoriza ou atribui peso para indicar o uso de grades. Isso tanto pode ser positivo, ao proporcionar liberdade de decisão ao enfermeiro, quanto pode ser uma limitação.

\section{Conclusão}

A tradução e adaptação do instrumento Evaluation of Siderail Usage para a língua 
portuguesa do Brasil obteve como produto um instrumento para avaliação do uso de grades em pacientes hospitalizados. O instrumento apresentou bons índices de validade de conteúdo. Sua validação na prática clínica dos enfermeiros pode auxiliar na tomada de decisão e avaliação dos pacientes quanto ao uso de grades, contribuindo, assim, para que as grades sejam utilizadas com uma avaliação adequada.

\section{Colaborações:}

1 - concepção, projeto, análise, e interpretação dos dados: Teresa Cristina Brasil Ferreira, Rosimere Ferreira Santana e Thalita Gomes do Carmo;

2 - redação do artigo, revisão crítica relevante do conteúdo intelectual: Márcia Veríssimo de Souza, Priscilla Alfradique de Souza e Thalita Gomes do Carmo;

3 - aprovação final da versão a ser publicada: Teresa Cristina Brasil Ferreira, Rosimere Ferreira Santana, Thalita Gomes do Carmo, Márcia Veríssimo de Souza e Priscilla Alfradique de Souza.

\section{Referências}

1. Guirguis-Blake JM, Michael YL, Perdue LA, Coppola EL, Beil TL. Interventions to Prevent Falls in Older Adults: Updated Evidence Report and Systematic Review for the US Preventive Services Task Force. JAMA. 2018 Apr;319(16):1705-16. DOI: 10.1001/jama.2017.21962

2. Slawomirski L, Auraaen A, Klazinga NS. The economics of patient safety: Strengthening a value-based approach to reducing patient harm at national level. OECD Health Working Papers. 2017;96:1-67. DOI: https://doi.org/10.1787/ 5a9858cd-en

3. Mitchell R, Faris M, Lystad R, Pulido DF, Norton G, Baysari $\mathrm{M}$, et al. Using the WHO International Classification of patient safety framework to identify incident characteristics and contributing factors for medical or surgical complication deaths. Appl Ergon. 2020;82:102920. DOI: 10.1016/j.apergo.2019.102920
4. Walter M, Pavão ALB, Martins M, Travassos C. The application of Iberoamerican study of adverse events (IBEAS) methodology in Brazilian hospitals. Int J Qual Health Care. 2018;30(6):480-5. DOI: 10.1093/intqhe/mzy055

5. Joint Commission Center. A complimentary publication of The Joint Commission. Inadequate hand-off communication. Sentinel Event Alert [Internet]. 2017 [cited $2020 \mathrm{Feb}$ 2];58:1-6. Available from: https://e-handoff.com/wp-content/uploads/ 2017/09/Joint-Commision-Handoff-CommunicationAlert.pdf

6. Tiensoli SD, Moreira MC, Morais SM, Matozinhos FP, Gomes FSL. Contexto de quedas notificadas em um hospital universitário. Rev baiana enferm. 2019;33:e32590. DOI:10.18471/ rbe.v33.32590

7. Ang SY, Aloweni FAB, Perera K, Wee SL, Manickam A, Lee $\mathrm{JH}$, et al. Physical restraints among the elderly in the acute care setting: Prevalence, complications and its association with patients' characteristics. Proc Singapore Healthc. 2015;24(3):137-43. DOI: $10.1177 / 2010105815596092$

8. Bellenger E, Ibrahim JE, Bugeja L, Kennedy B. Physical restraint deaths in a 13-year national cohort of nursing home residents. Age Ageing. 2017;46(4):688-93. DOI: 10.1093/ageing/afw246

9. Shorr RI, Staggs VS, Waters TM, Daniels MJ, Liu M, Dunton N, et al. Impact of the HospitalAcquired Conditions Initiative on Falls and Physical Restraints: A Longitudinal Study. J Hosp Med. 2019;9(18):E31-E36. DOI: 10.12788/ jhm.3295

10. Capezuti E, Maislin G, Strumpf N, Evans L. Side rail use and bed-related falls outcomes among nursing home residents. J Am Geriatr Soc. 2002;50(1):90-6. DOI: 10.1046/j.1532-5415.2002.50013.x

11. Brem H, Maggi J, Niermen D, Rolnitzky L, Bell D, Rennert R, et al. High cost of stage IV pressure ulcers. Am J Surg. 2010;200(4):473-7. DOI: 10.1016/j.amjsurg.2009.12.021

12. Sireci SG, Yang Y, Harter J, Ehrlich EJ. Evaluating Guidelines For Test Adaptations: A Methodological Analysis of Translation Quality. J Cross Cult-Psychol. 2006;37(5):557-67. DOI: $10.1177 / 0022022106290478$

13. Cassepp-Borges V, Balbinotti MAA, Teodoro MLM. Tradução e validação de conteúdo: Uma proposta 
para a adaptação de instrumentos. In: Pasquali L. Instrumentação psicológica: Fundamentos e práticas. Porto Alegre: Artmed; 2010. p. 506-20.

14. Guillemin F, Bombardier C, Beaton D. Cross-cultural adaptation of health-related quality of life measures: literature review and proposed guidelines. J Clin Epidemiol. 1993;46(12):1417-32. DOI: 10.1016/0895-4356(93) 90142-n

15. Fitzpatrick R. The International Assessment of Health-related Quality of Life: Theory, Translation, Measurement and Analysis. J Med Ethics. 1996;22(4):248-9. DOI: http://dx.doi.org/10.1136/ jme.22.4.248

16. Fortes CPDD, Araújo APQC. Check list para tradução e Adaptação Transcultural de questionários em saúde. Cad saúde colet. 2019;27(2):202-9. DOI: 10.1590/1414-462x201900 020002

17. Torlig EGS, Resende Junior PC. Validação de Instrumento de Coleta de Dados: Experiência com o Coeficiente de Validação de Conteúdo (CVC) e Proposição de uma nova Abordagem para Pesquisas Qualitativas. In: Congresso Ibero-Americano em Investigação Qualitativa, 8, 2019, Lisboa, PT. Atas (on-line). Lisboa: CIAIQ; 2019. Investigação Qualitativa em Ciências Sociais, v. 3 p. 50-9. [cited 2020 Feb 5]. Available from: https://proceedings.ciaiq.org/ index.php/CIAIQ2019/article/view/1984/1920

18. Sarlet GBS, Caldeira C. O consentimento informado e a proteção de dados pessoais de saúde na internet: uma análise das experiências legislativas de Portugal e do Brasil para a proteção integral da pessoa humana. Civilistica. com [Internet]. 2019 [cited 2020 Feb 8];8(1):1-27.
Available from: https://civilistica.emnuvens.com. $\mathrm{br} / \mathrm{redc} /$ article/view/411

19. Huynh D, Lee ON, An PM, Ens TA, Mannion CA. Bedrails and Falls in Nursing Homes: A Systematic Review. Clin Nurs Res. 2021;30(1):5-11. DOI: 10.1177/1054773820907805

20. Rosa VPP, Cappellari FCBD, Urbanetto JS. Análise dos fatores de risco para queda em idosos institucionalizados. Rev bras geriatr gerontol. 2019;22(1):e180138. DOI: 10.1590/198122562019022.180138

21. Medicines and Healthcare Products Regulatory Agency. Safe use of bed rails [Internet]. London (UK); 2021. [cited 2020 Aug 9]. Available from: https://assets.publishing.service. gov.uk/government/uploads/system/uploads/ attachment_data/file/880287/FINAL_Safe_ Use_of_Bed_Rails_v3.pdf

22. Ferreira TCB, Santana RF, Carmo FGD, Souza MVD, Souza LMDS. Instrumentos para avaliação do uso de grades laterais do leito: segurança ou contenção? Nurs [Internet]. 2019 [cited 2020 Aug 12];22(254):3052-59. Available from: http://www.revistanursing.com.br/revistas/ 254/pg35.pdf

23. Shuman CJ, Montie M, Hoffman GJ, Powers KE, Doettl S, Anderson CA, et al. Older Adults' Perceptions of Their Fall Risk and Prevention Strategies After Transitioning from Hospital to Home. J Gerontol Nurs. 2019;45(1):23-30. DOI: 10.3928/00989134-201901 $02-04$

Recebido: 9 de janeiro de 2021

Aprovado: 20 de junho de 2020

Publicado: 22 de julho de 2021

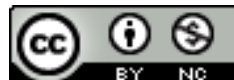

A Revista Baiana de Enfermagem utiliza a Licença Creative Commons - Atribuição-NãoComercial 4.0 Internacional. https://creativecommons.org/licenses/by-nc/4.0/

Este artigo é de acesso aberto distribuído sob os termos da Licença Creative Commons (CC BY-NC).

Esta licença permite que outros remixem, adaptem e criem a partir do seu trabalho para fins não comerciais. Embora os novos trabalhos tenham de lhe atribuir o devido crédito e não possam ser usados para fins comerciais, os usuários não têm de licenciar esses trabalhos derivados sob os mesmos termos. 